\title{
Nrf2 Activation Induced by Sirt1 Ameliorates Acute Lung Injury After Intestinal Ischemia/Reperfusion Through NOX4-Mediated Gene Regulation
}

\author{
DongDong Chai Lei Zhang SiWei Xi YanYong Cheng Hong Jiang Rong Hu \\ Department of Anesthesiology, Shanghai Ninth People's Hospital, Shanghai JiaoTong University, School \\ of Medicine, Center for Specialty Strategy Research of Shanghai Jiao Tong University China Hospital \\ Development Institute, Shanghai, China
}

\section{Key Words}

$\mathrm{Nrf2} \cdot$ Sirt1 $・$ NOX4 $・$ Migration $・$ Angiogenesis

\begin{abstract}
Background/Aims: Nuclear erythroid 2-related factor-2 (Nrf2) is a major stress-response transcription factor that has been implicated in regulating ischemic angiogenesis. We investigated the effects of Nrf2 in regulating revascularization and modulating acute lung injury. Methods: The expression of Nrf2 and sirtuin1 (Sirt1) was assessed in lung tissue by western blotting and immunofluorescence staining after intestinal ischemia/reperfusion (IIR) in Nrf2-/- and wild-type (WT) mice. The involvement of Nrf2 in angiogenesis, cell viability, and migration was investigated in human pulmonary microvascular endothelial cells (PMVECs). Additionally, the influence of Nrf2 expression on NOX pathway activation was measured in PMVECs after oxygen-glucose deprivation/reoxygenation. Results: We found activation and nuclear accumulation of Nrf2 in lung tissue after IIR. Compared to IIR in WT mice, IIR in Nrf2-/- mice significantly enhanced leukocyte infiltration and collagen deposit, and inhibited endothelial cell marker CD31 expression. Nrf2 upregulation and translocation into the nucleus stimulated by Sirt1 overexpression exhibited remission of histopathologic changes and enhanced CD31 expression. Nrf2 knockdown repressed non-phagocytic cell oxidase 4 (NOX4), hypoxia-inducible factor (HIF-1 $\alpha$ ) and vascular endothelial growth factor (VEGF) expression after IIR. Nrf2 upregulation by Sirt1 enhances NOX4, HIF-1 $\alpha$ and VEGF expression after IIR in WT mice. Furthermore, Nrf2 knockdown suppressed cell viability, capillary tube formation and cell migration in PMVECs after oxygen-glucose deprivation/reoxygenation and also inhibited NOX4, HIF-1 and VEGF expression. Moreover, NOX4 knockdown in PMVECs decreased the levels of VEGF, HIF-1 $\alpha$ and angiogenesis. Conclusion: Nrf2 stimulation by Sirt1 plays an important role in sustaining angiogenic potential through NOX4-mediated gene regulation.

D.-D. Chai and L. Zhang contributed equally to this work.




\section{Introduction}

Intestinal ischemia/reperfusion (IIR) injury is an intraoperative complication that can give rise to secondary vascular disease and a number of associated physiological conditions, which relate predominantly to the release of cytotoxic substances from ischemic tissues and inflammatory mediators [1]. As a consequence, IIR may lead to sepsis, systemic inflammatory response syndrome and multiple organ dysfunction syndrome [2]. IIR is also known to cause acute lung injury which in turn leads to a condition known as acute respiratory distress syndrome and contributes to the high mortality associated with IIR [35]. The exact participation of IIR in acute lung injury is not clearly understood but is believed to involve the initiation of cell apoptosis through a complex interaction between reactive oxygen species, cytokines and inflammatory mediators [6].

$\mathrm{Nrf} 2$ is known to activate various antioxidant responsive element (ARE)-dependent genes in response to oxidative stress [7, 8]. Under basal conditions Nrf2 is bound to KEAP1 (Kelch-like ECH-associated protein 1) in the cytosol, however, on exposure to oxidative stress, Nrf2 disassociates from KEAP1 and translocates into the nucleus where it enhances the transcription of target antioxidant genes through AREs, thereby increasing cytoprotection [9]. Nrf2 has also been implicated in regulating ischemic angiogenesis in the retina and other tissue beds $[10,11]$. Nrf2 is thought to confer a protective role in lung damage associated with oxidative stress as inflammation and injury are increased when it is deficient [12]. In an acute lung injury model, hyperoxia-induced NOX4 was found to be reduced in Nrf2-/- mice as Nrf2 regulates NOX4 expression [13]. NOX4 is believed to be regulated by three potential ARE sites, and the deletion of two of these sites was found to abolish hyperoxia-induced NOX4 promoter activity [14]. Nrf2 was also found to bind to the NOX4 promoter in response to hyperoxia [14].

Sirt1 is a nicotinamide adenine dinucleotide (NAD+)-dependent deacetylase protease which is thought to be involved in many regulatory processes through deacylation and interaction with several transcription factors and signalling molecules including those involved in neovascularisation [15]. Upregulated Sirt1 expression was found to downregulate FOXO3 acetylation to confer a protective role in IIR acute lung injury by modulating the effects of downstream anti-oxidative and anti-apoptotic factors [1]. Furthermore, upregulating levels of Sirt1 has also been found to increase the nuclear translocation of Nrf2 which in turn inhibits mitogen-activated protein kinase phosphorylation and offers protection against cellular damage caused by oxidative stress [16-18].

In this work, we investigate the Nrf2/Sirt1 pathway in regulating revascularization and modulating acute lung injury after IIR in Nrf2-deficient mice and in Nrf2 knockdown human pulmonary microvascular endothelial cells (PMVECs) after oxygen-glucose deprivation/ reoxygenation $(\mathrm{OGD} / \mathrm{R})$.

\section{Materials and Methods}

\section{Experimental model}

Sixty eight-week-old C57BL/6J mice and 48 eight-week-old Nrf2 knockout (Nrf2-/-) mice with the same genetic background (provided by the RIKEN Bio-Resource Centre through the National Bio-Resource Project, MEXT, Japan) were used to conduct the in vivo experiments. Mice were kept at a controlled temperature $\left(21 \pm 2^{\circ} \mathrm{C}\right)$ and humidity $(60 \pm 5 \%)$ on a 12-h light/dark cycle. They were fed a standard mice chow and water ad libitum. All experiments were conducted in conformity with NIH guidelines and approved by the Ethical Committee of Shanghai Ninth People's Hospital for Animal Research.

Lung tissue overexpression of Sirt1 by adenovirus infection in vivo.

Adenoviral-mediated gene delivery was performed as previously described [19]. Adenoviral vectors (3 $\times 10^{10}$ pfu per mouse) expressing Sirt1 were administered by direct injection to the lungs of anaesthetized (sodium pentobarbital) mice by intratracheal instillation. Data were expressed as percentage activity. 


\section{Cellular Physiology Cell Physiol Biochem 2018;46:781-792

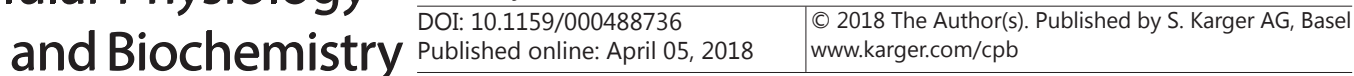

\section{Mice IIR model}

Mice were randomly assigned into four groups: ( $\mathrm{n}=6 /$ group): sham; IIR; control vector + IIR; and Sirt1 vector + IIR. They were then anaesthetized with sodium pentobarbital (50 mg/kg, i.p.) and allowed to breathe spontaneously during surgery. The abdominal wall of anaesthetized mice was opened by midline incision. The superior mesenteric artery was exposed and clamped by an aneurysm clip. The blood flow to the intestine was interrupted for 90 minutes then released to re-establish blood flow. The sham group underwent an identical operation except for the clamping of the superior mesenteric artery. All mice were resuscitated with an intraperitoneal injection of normal saline $(1.0 \mathrm{ml})$ after surgery. Animals were sacrificed after $6 \mathrm{~h}$ of reperfusion and tissues were harvested. Tissues were snap frozen in liquid nitrogen and stored at $-80^{\circ} \mathrm{C}$ until analysis.

\section{Cell isolation}

Lung tissue was dissociated by pipetting up and down in $10 \mathrm{ml}$ of sort buffer (D-PBS with 2\% FBS, $1 \mathrm{mM} \mathrm{EDTA}$ ) and $50 \mathrm{U} / \mathrm{ml}$ DNAse. The sample was incubated for $10 \mathrm{mins}$ at $37^{\circ} \mathrm{C}$ with rocking and then transferred through $100 \mu \mathrm{m}, 70 \mu \mathrm{m}$, and $40 \mu \mathrm{m}$ cell strainers into $50 \mathrm{ml}$ tubes. The filtered suspension was transferred to a $15-\mathrm{mL}$ tube and spun for $5 \mathrm{~min}$ at $550 \times \mathrm{g}$ at $4{ }^{\circ} \mathrm{C}$ to pellet cells. The supernatant was removed and the cells resuspended in $10 \mathrm{ml}$ of sort buffer and left to recover by incubation with shaking for one hour at $37^{\circ} \mathrm{C}$.

\section{Histological examination}

Ischemia-reperfusion injury was determined in 4- $\mu \mathrm{m}$ haematoxylin and eosin (HE) stained paraffinembedded sections. Acute lung injury was scored using a five-point scale according to combined assessments of alveolar congestion, haemorrhage, oedema, and infiltration of inflammatory cells in the airspace or vessel wall. A 0-4 (minimal to maximal severity) scoring system was used in a blinded manner. Lung fibrosis was measured by the quantitative analysis of Masson's Trichrome positive-staining area with software Image Pro-Plus 6.0. Images were captured with a digital camera (Optronics DEI-470; Goleta, CA) connected to a microscope.

The degree of lung injury was assessed using a scoring system. According to this scoring system, oedema of the alveoli and mesenchyme, intra-alveolar inflammatory cell infiltrates, alveolar haemorrhage and atelectasis were graded on a scale between 0 and 4 . The grades were as follows: 0 , normal, $<15 \%$ of space occupied by tissue and $>85 \%$ occupied by alveolar space; $1,15 \%-25 \%$ of space occupied by tissue and $75 \%-85 \%$ occupied by alveolar space; $2,25 \%-50 \%$ occupied by tissue and $50 \%-75 \%$ occupied by alveolar space; $3,50 \%-75 \%$ occupied by tissue and $25 \%-50 \%$ occupied by alveolar space; and $4,75 \%-$ $100 \%$ occupied by tissue and $0 \%-25 \%$ occupied by alveolar space.

\section{Quantification of lung collagen}

After severing the abdominal aorta, the pulmonary vasculature was perfused with sterile saline (15 $\mathrm{ml}$ ) and left lungs were excised at the hilum, homogenised, and then incubated in $65 \%$ trichloroacetic acid (Fisher Scientific, Pittsburgh, PA) on ice. Following centrifugation and aspiration of the supernatant, the tissue pellet was resuspended in $12 \mathrm{~N} \mathrm{HCl}$ and baked at $110^{\circ} \mathrm{C}$ overnight. The denatured tissue was then reconstituted in $\mathrm{ddH}_{2} \mathrm{O}$, and aliquots $(200 \mu \mathrm{l}$ ) were incubated with $1.4 \%$ chloramine T (500 $\mu \mathrm{l}$; SigmaAldrich, St Louis, MO) in 10\% isopropanol and $0.5 \mathrm{M}$ sodium acetate for $20 \mathrm{~min}$ at room temperature. 1 M 4-(dimethylamino)-benzaldehyde (500 $\mu$; Sigma-Aldrich) was then added, and the suspension was incubated for $15 \mathrm{~min}$ at $65^{\circ} \mathrm{C}$. Absorbance at $540 \mathrm{~nm}$ was measured in triplicate for each sample. A standard curve was generated simultaneously using $0-500 \mu \mathrm{g} / \mathrm{ml}$ trans-4-hydroxy-L-proline (Sigma-Aldrich), starting with the chloramine reaction.

\section{Pulmonary oedema}

The level of pulmonary oedema was tested using a wet/dry ratio. A median sternotomy was performed after completing reperfusion. The lung lobe was cut from the pleural cavity and the right lung was placed in a drying oven $\left(90^{\circ} \mathrm{C}\right)$ for $24 \mathrm{~h}$ after it was weighed. The right lung was weighed again after drying was accomplished. The wet weight of lung was divided by the dry weight to calculate the wet/dry ratio.

The middle lobes of the right lungs were weighed ( weight $_{\text {wet }}$ ) immediately using a precision balance (Mettler-Toledo, Schweiz, GmbH, Greifensee, Switzerland), and re-weighed (weight ${ }_{\text {dry }}$ ) following incubation 


\section{Cellular Physiology Cell Physiol Biochem 2018;46:781-792 \begin{tabular}{ll|l} 
DOI: 10.1159/000488736 & O 2018 The Author(s). Published by S. Karger AG, Basel \\
www.karger.com/cpb
\end{tabular} \\ Chai et al.: Nrf2 Activation Ameliorates ALI}

at $95^{\circ} \mathrm{C}$ in an oven (876-1 Vacuum Drying Oven; Nantong Science Instrument Factory, Nantong, China) for $24 \mathrm{~h}$. The $\mathrm{w} / \mathrm{d}$ ratio was calculated as follows: $\mathrm{w} / \mathrm{d}=$ weight $_{\text {wet }} /$ weight $_{\text {dry }}$.

\section{Immunofluorescence and immunohistochemistry}

Lung specimens were fixed in 10\% formalin, dehydrated in alcohol, embedded in paraffin, and then cut into 5 - $\mu \mathrm{m}$ thickness sections and mounted. Sections were deparaffinized with xylene, and heat-mediated Ag retrieval was performed. All sections were incubated overnight at $4^{\circ} \mathrm{C}$ with anti-CD31 (Abcam, Cambridge, UK), Nrf2 (Abcam) and NOX4 (Abcam) primary antibody. The following day, slides were incubated with anti-mouse Alexa Fluor 488 and anti-rabbit Alexa Fluor 555 (Life, Eugene, OR) secondary antibody at $37^{\circ} \mathrm{C}$ for $1 \mathrm{~h}$. DAPI was used for nuclear staining. For immunohistochemistry of CD31, dewaxed and hydrated lung tissue sections were treated with $3 \%$ hydrogen peroxide solution for $10 \mathrm{~min}$, rinsed with PBS, and incubated with anti-CD31 antibody (1:100 working dilution, Millipore, Temecula, CA) at $4^{\circ} \mathrm{C}$ overnight. The lung sections were then incubated with secondary antibody at $37^{\circ} \mathrm{C}$ for $15 \mathrm{~min}$, exposed to streptavidinhorseradish peroxidase at $37^{\circ} \mathrm{C}$ for $15 \mathrm{~min}$, and stained with a DAB substrate kit (Vector Laboratories, Burlingame, CA) and Meyer haematoxylin.

Oxygen-glucose deprivation and reoxygenation of human pulmonary microvascular endothelial cells

Human PMVECs were purchased from Science Cell Research Laboratories (Catalogue Number: 3000). To perform OGD/R, cells were cultured in glucose-free DMEM (11966-025; Invitrogen). They were then washed in PBS supplemented with $0.5 \mathrm{mM} \mathrm{CaCl}$ and $1 \mathrm{mM} \mathrm{MgCl}_{2}$ and placed in an anaerobic chamber $(5 \%$ $\mathrm{CO}_{2}, 95 \% \mathrm{~N}_{2}$; Memmert; Schwabach, Germany) to induce OGD. After $8 \mathrm{~h}$, the medium was replaced with ECM and the plates were grown in a normoxic chamber $\left(37^{\circ} \mathrm{C}, 5 \% \mathrm{CO}_{2}\right)$ for $24 \mathrm{~h}$ reoxygenation.

\section{Cord formation assay}

Growth factor-reduced Matrigel ( $300 \mu \mathrm{l} ; \mathrm{BD}$ ) was added to each well of a 24-well plate and allowed to polymerise for $2 \mathrm{~h}$ at $37^{\circ} \mathrm{C}$. A total of $1 \times 10^{5}$ cells were preincubated with serum-free EGM medium for $1 \mathrm{~h}$ and then seeded. Cells were viewed, photographed, and quantified using a Leica microscope (Leica), ImageJ and Metamorph software (Molecular Devices), respectively, after $6 \mathrm{~h}$ incubation at $21 \%$ or $1 \% \mathrm{O}_{2}$. The number of cords was determined by counting cytoplasmic extensions, and branch points; visible lumens, and total cord length were also determined. At least three fields per well were examined, and each experimental condition was tested in triplicate.

\section{Proliferation screening and cell proliferation assays}

Viable cell number was estimated using a Cell Counting Kit-8 (Dojindo, Kumamoto, Japan) assay. To measure the proliferative activity of cells in 96-well microplates, CCK-8 was added (10 $\mu \mathrm{l} /$ well) and incubated for $2 \mathrm{~h}$. Absorbance was measured at $450 \mathrm{~nm}$ using a microplate reader (Molecular Devices) with a reference wavelength of $650 \mathrm{~nm}$.

\section{Cell migration assays}

Cells were resuspended in culture medium without serum and seeded at densities of $2 \times 10^{4}$ cells/ well in 24-well Transwell inserts ( $8 \mu \mathrm{m}$ pores, CorningCostar, Rochester, NY, USA) then incubated for 24 $\mathrm{h}$ at $37^{\circ} \mathrm{C}$. Migrated cells remaining on the bottom surface were Giemsa stained and counted under a light microscope (Leica, Germany).

\section{Transient transfection of PMVECs with Sirt1 vector}

The transfection of PMVECs with the Sirt1 lentiviral vector was carried out as described previously [15]. PMVECs were plated in 6-well plates at a density of $2 \times 10^{5}$ per well and then were infected with lentiviral vectors at a multiplicity of infection (MOI) of 20. The gene transduction efficiency of lentiviral vectors was indicated by GFP or RFP expression and detected by flow cytometry.

\section{Cell immunofluorescence microscopy}

PMVECs were grown on coverslips to 95\% confluence in EGM-2 complete medium as indicated. Coverslips were rinsed with phosphate-buffered saline (PBS) and treated with $3.7 \%$ formaldehyde in PBS at room temperature for 20 min. After washing with PBS, coverslips were incubated in blocking 


\section{Cellular Physiology Cell Physiol Biochem 2018;46:781-792

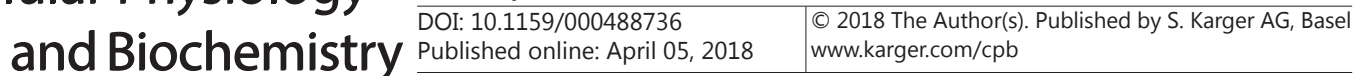

Chai et al.: Nrf2 Activation Ameliorates ALI

buffer [1\% bovine serum albumin in Tris-buffered saline-Tween-20 (TBST)] for $1 \mathrm{~h}$. Cells were subjected to immunostaining with total NOX4 antibody or Nrf2 antibody (1: 200 dilution) for $1 \mathrm{~h}$ and washed three times with TBST, followed by staining with Alexa Fluor 488 (1:200 dilution in blocking buffer) or Alexa Fluor 555 for $1 \mathrm{~h}$. After washing at least three times with TBST, the coverslips were mounted using commercial mounting medium (Kirkegaard and Perry Laboratories, Gaithersburg, MD) and were examined by immunofluorescence microscopy with a Hamamatsu digital camera using a 60x oil immersion objective and MetaVue software.

\section{RNA extraction and real-time PCR}

Total RNA was extracted using an RNeasy minikit (QIAGEN, Hamburg, Germany). For real-time PCR, RNA $(2 \mu \mathrm{g})$ was transcribed reversely to a template of complementary DNA (40 ng). The amplification cycling reactions ( 40 cycles) were performed as follows: $5 \mathrm{~s}$ at $95^{\circ} \mathrm{C}$ and $30 \mathrm{~s}$ at $60^{\circ} \mathrm{C}$. Relative quantification values of the target genes were standardised according to the comparative threshold cycle $(2-\Delta \Delta \mathrm{CT})$. VEGF

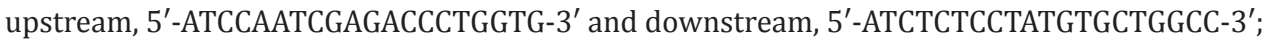

HIF-1 $\alpha$ upstream, $5^{\prime}$-TAGAGTCAGCAACGTGGAAG- $3^{\prime}$ and downstream, $5^{\prime}$-TATCGAGGCTGTGTCGACTG-3'.

\section{Preparation of nuclear extracts}

Nuclear extracts were prepared from PMVECs by a method described previously [14]. Briefly, cells were collected by scraping in ice-cold PBS containing phosphatase inhibitors and pelleted by centrifuging at $1000 \mathrm{x}$ g for $5 \mathrm{~min}$. The pellet was resuspended in $500 \mu \mathrm{l}$ of $1 \mathrm{x}$ hypotonic buffer and incubated on ice for $15 \mathrm{~min}$, followed by the addition of detergent ( $25 \mu \mathrm{l})$ and high-speed vortexing for $30 \mathrm{~s}$. The suspension was then centrifuged $\left(14,000 \mathrm{x}\right.$ g for $20 \mathrm{~min}$ ) at $4^{\circ} \mathrm{C}$; the nuclear pellet was resuspended in lysis buffer (50 $\mu \mathrm{l})$ and incubated on ice for $15 \mathrm{~min}$. This suspension was centrifuged (14, $000 \mathrm{xg}$ ) for a further $10 \mathrm{~min}$ and aliquots of the supernatant (nuclear extract) were stored at $-80^{\circ} \mathrm{C}$ for further analysis. Protein content in the nuclear extract was quantified by BCA protein assay.

\section{Western blot analysis}

Western blotting was carried out by a standard protocol. Proteins (30 $\mu \mathrm{g})$ in the total cell lysate or lung tissue were separated by SDS-PAGE and transferred to nitrocellulose membranes. Bands were visualised by chemiluminescence (ECL; Amersham), followed by exposure to x-ray film (RX-U; Fujifilm) and densitometrically quantified using ImageJ (National Institutes of Health).

\section{Transient transfection of PMVECs with NOX4 siRNA}

Transfection of PMVECs with NOX4 siRNA was carried out as described previously [14]. Briefly, PMVECs were grown to $~ 70 \%$ confluence in 6-well plates and transfected with Fugene HD Transfection Reagent (Roche Applied Science, IN, USA) containing NOX4 wild-type (1 $\mu$ g cDNA), scrambled siRNA (50 $\mathrm{nM}$ ) or siRNA for NOX4 (50 nM) in serum-free EBM-2 medium. After $3 \mathrm{~h}$ transfection, the serum-free media was replaced by $1 \mathrm{ml}$ of fresh complete EGM-2 medium containing 10\% FBS and growth factors. Cells were cultured for an additional $48 \mathrm{~h}$ before analysis by western blotting.

\section{Statistical analysis}

All data are presented as the mean \pm SD (standard error of the mean). Data were compared using oneway ANOVA followed by the Student's $t$-test for unpaired data with Bonferroni correction. Square roots of tissue cell counts were compared using one-way ANOVA.

\section{Results}

Nrf2 activation and nuclear accumulation after intestinal ischemia/reperfusion

We investigated the effects of IIR on the activation and location of Nrf2 in WT mice by immunofluorescence staining (Fig. 1A, B) and western blotting (Fig. 1C). We found that Nrf2 significantly accumulates in the nucleus in lung tissue after IIR compared with sham mice. We then investigated the effects of deleting Nrf2 on lung injury modulation and angiogenesis following IIR. Compared to WT mice, IIR in Nrf2-/- mice led to significantly enhanced 
Fig. 1. Modulation of lung injury after intestinal ischemia/reperfusion (IIR) is impaired in Nrf2-deficient mice. Mice were subjected to IIR and lung tissues were harvested. (A) Increased expression and nuclear translocation of Nrf2 after IIR were determined by immunofluorescence staining. (B) Percentage of specimens showing Nrf2 expression. Quantification of immunofluorescence staining. (C) Western blotting of the expression and nuclear translocation of Nrf2 after IIR. (D) Representative HE staining of lung tissues of mice following IIR.. (E) Histopathological mean lung injury scores were higher in Nrf2-/- following IIR. The wet/dry ratio of lung tissue in WT was lower than in Nrf2-/-mice. More collagen was deposited in the lungs of Nrf2-/- mice following IIR. $\left(^{*} \mathrm{p}<0.05\right.$; ** $\mathrm{p}<0.001$ vs. respective control).

leukocyte infiltration $(p<$ 0.05 ) and collagen deposit ( $p$ $<0.05$ ) (Fig. 1D, E). Moreover, lung injury score was higher in Nrf2-/- mice than in WT mice. In addition, the endothelial cell marker CD31 expression was inhibited $(p<0.05)$ (Fig. 2). These results indicate that $\mathrm{Nrf} 2$ is translocated into the nucleus following IIR and that the modulation of lung injury is impaired in Nrf2-deficient mice and angiogenesis is inhibited.

\section{Sirt1 induced Nrf2 upregulation and translocation into the nucleus}

WT mice were given an intravenous injection of adenovirus vector expressing Sirt1 before being subjected to IIR. Western blotting and immunofluorescence staining show that Sirt1 overexpression induces Nrf2 upregulation and translocation into the nucleus in WT mice (Fig. 3A-C). Furthermore, Sirt1 overexpression attenuates lung injury after IIR through improving

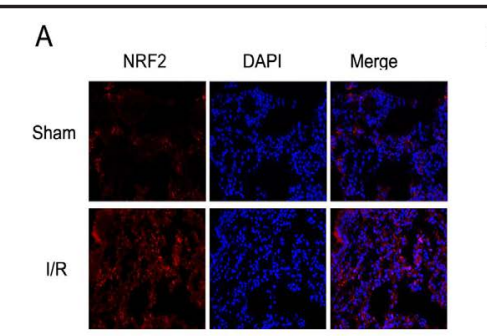

B

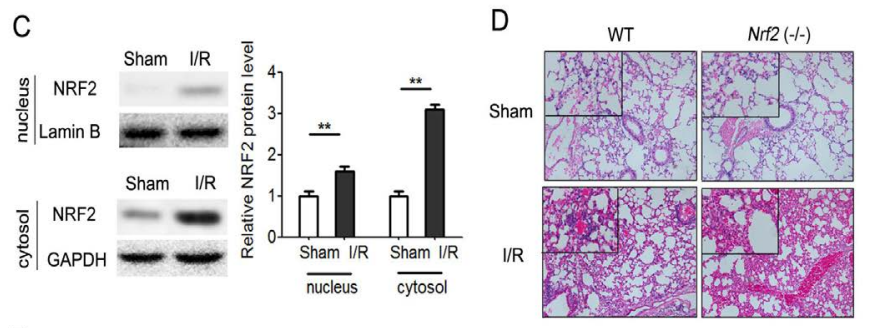

E
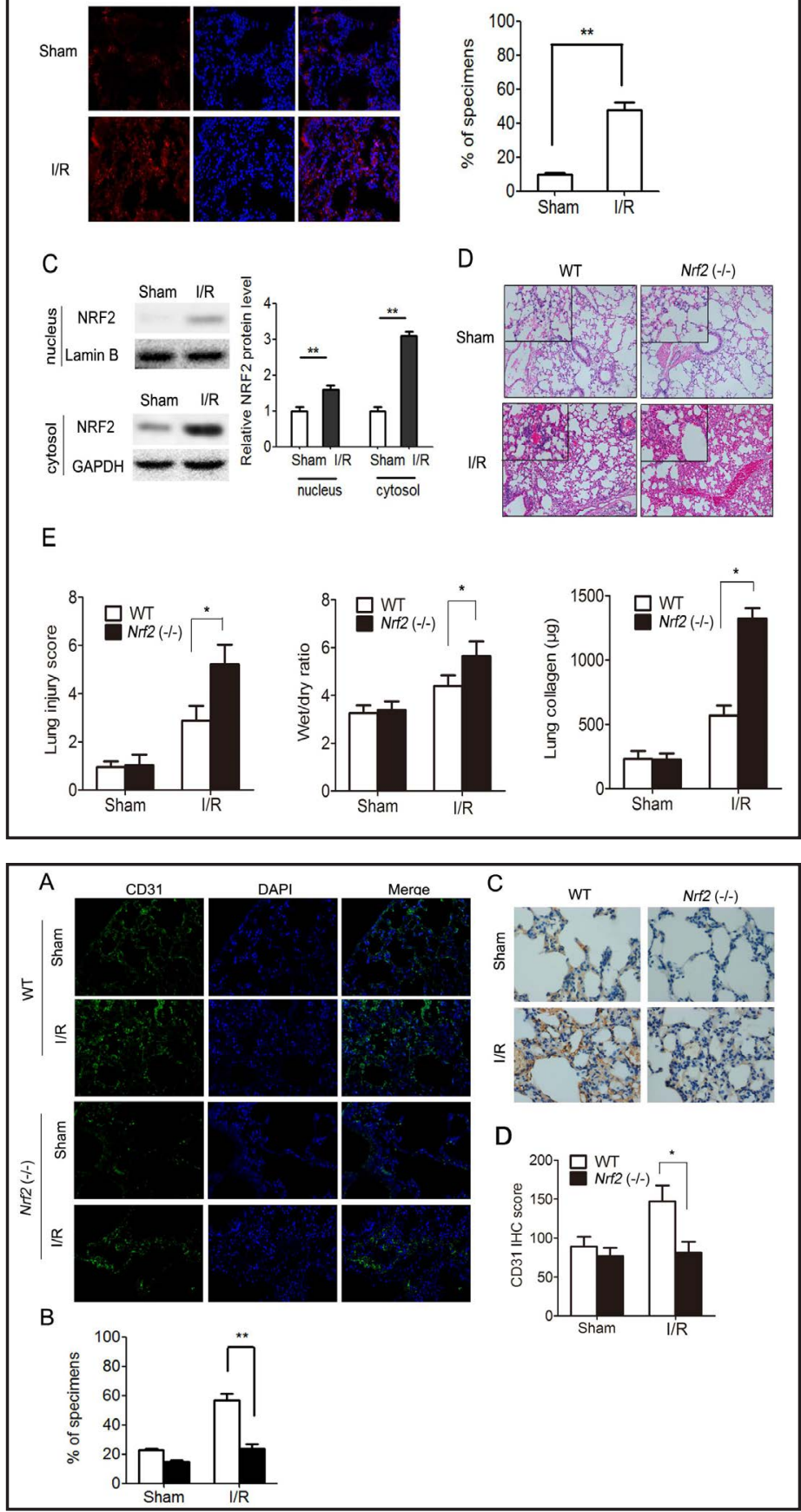

Fig. 2. Nrf2 deficiency inhibited angiogenesis. (A, B) CD31 was increased in WT mice compared with Nrf2-/- mice following IIR. (C) Representative photographs showing CD31 staining in lung tissues of WT and Nrf2-/- mice following IIR. (D) Column plots for CD31 IHC staining scores in WT and Nrf2-/- mice following IR. $\left({ }^{*} \mathrm{p}<0.05\right.$; ${ }^{* *} \mathrm{p}<$ 0.001 vs. respective control). 


\section{Cellular Physiology

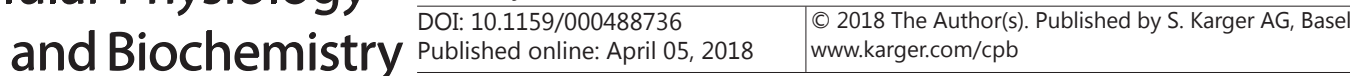

Fig. 3. Sirt1 overexpression induces Nrf2 upregulation and translocation into the nucleus in WT mice and attenuates lung injury after IIR through improving angiogenesis. WT mice were given an intravenous injection of adenovirus vector before being subjected to IIR. Lung tissues were harvested 7 days after perfusion to enable the detection of the Sirt1 protein (A). The increased expression and nuclear translocation of Nrf2 in mice overexpressing Sirt1 were determined by western blotting (B) and immunofluorescence staining (C). Representative HE staining of lung tissues of mice following injection of the Sirt1 expression vector (D). Histopathological mean lung injury scores in mice following injection of the Sirt1 expression vector (E). Mice overexpressing Sirt1 had a lower wet/dry lung ratio (F). Less collagen was deposited in the lungs of WT mice following injection of the Sirt1 expression vector $(G)$. (H) CD31 was increased in mice following injection of the Sirt1 expression vector compared with that of the control vector in WT mice following IIR. (I) Representative photographs showing CD31 staining. (J) Column plots for CD31 IHC staining scores. Scores are increased in mice overexpressing $\operatorname{Sirt} 1\left(^{*} \mathrm{p}<0.05\right.$; ${ }^{* *} \mathrm{p}<0.001$ vs. respective control).

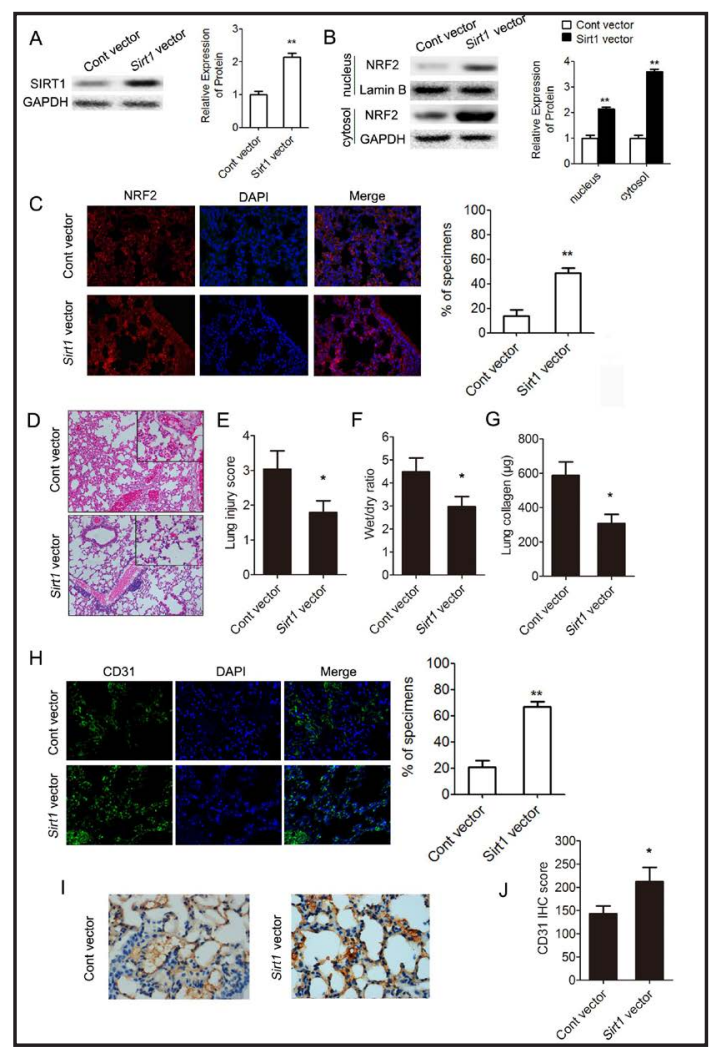

angiogenesis (Fig. 3D-F). More collagen was deposited in the lungs of WT mice that received the control vector than in WT mice injected with the Sirt1 expression vector $(p<0.05)$ (Fig. 3G). Mice overexpressing Sirt1 also exhibited remission of histopathologic changes and enhanced endothelial cell marker CD31 expression $(p<0.05)$ (Fig. 3H, J). To further analyse the involvement of Nrf2 in the mechanism of angiogenesis, we investigated NOX pathway activation through measuring NOX4 levels. By using immunofluorescence staining we found that Nrf2 knockdown repressed levels of NOX4 (Fig. 4A, B) and also repressed levels of hypoxia-inducible factor (HIF)-1 $\alpha$ and vascular endothelial growth factor (VEGF) expression after IIR (Fig. 4C, D). Whereas Sirt1 overexpression enhances NOX4 (Fig. 4E, F), HIF-1 $\alpha(p<$ $0.05)$ and VEGF ( $p<0.05)$ expression after IIR in wild-type mice (Fig. 4G, H). These results indicate that Sirt1 overexpression could induce Nrf2 upregulation and translocation into the nucleus in WT mice and attenuates lung injury after IIR through improving angiogenesis. Whereas, deleting Nrf2 in mice affects angiogenesis and NOX4-mediated gene regulation and consequently leads to the repression of HIF-1 $\alpha$ and VEGF.

Sirt1/Nrf2 increases angiogenesis in human PMVECs through NOX4-mediated regulation

To further investigate Nrf2/Sirt1 regulation we assessed the deletion of Nrf2 and overexpression of Sirt1 in human PMVECs. PMVECs were transfected with Nrf2 siRNA and exposed to OGD/R for $24 \mathrm{~h}$. Western blotting revealed that Nrf2 was absent from the nucleus (Fig. 5A) and cell viability, detected by CCK-8 at different time intervals following OGD/R, was reduced (Fig. 5B). Angiogenesis ( $p<0.001$ vs. control) and migration ( $p<0.001$ vs. control) were also reduced in the Nrf2 siRNA PMVECs (Fig. 5C, D). We transfected PMVEC cells with the Sirt1 vector and exposed them to OGD/R for $24 \mathrm{~h}$. Overexpression of Sirt1 induces Nrf2 upregulation and translocation into the nucleus and results in the nuclear accumulation of Nrf2 over time (Fig. 6A, B) and attenuates the reduction of survival, network formation, migration, and adhesion in PMVECs (Fig. 6C-F). We also assessed the involvement of the NOX 


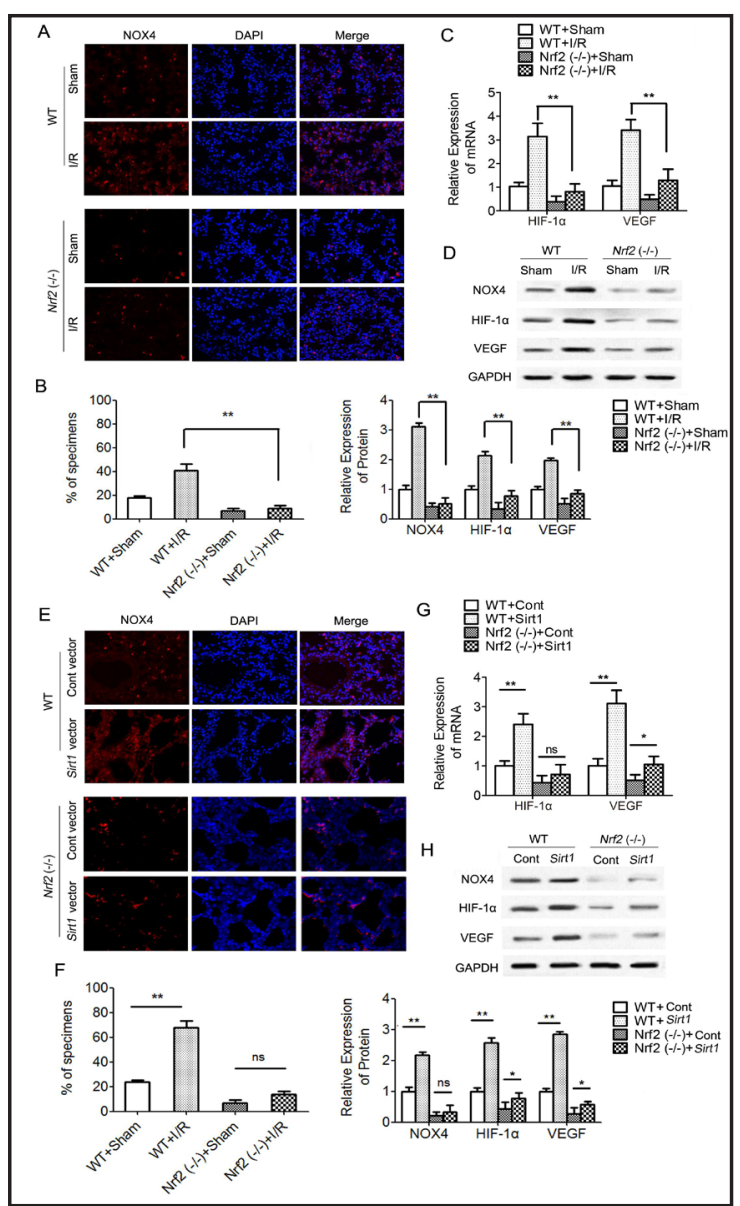

Fig. 4. Nrf2 deletion may affect angiogenesis through NOX4-mediated gene regulation. (A, B) Immunofluorescence staining of NOX4 in the lung tissue of WT and Nrf2-/- mice following IIR. (C, D) RTPCR and western blot analysis of HIF-1 $\alpha$ and VEGF regulated by NOX4. (E, F) Immunofluorescence staining of NOX4 in lung tissue specimens of WT and Nrf2-/- mice injected with Sirt1 vector after IIR. (G, H) RT-PCR and western blot analysis of HIF- $1 \alpha$ and VEGF. Regulation of Nrf2 by Sirt1 increased NOX4 and downstream protein expression. $\left({ }^{*} \mathrm{p}<0.05\right.$; ${ }^{* *} \mathrm{p}<0.001$ vs. respective control).

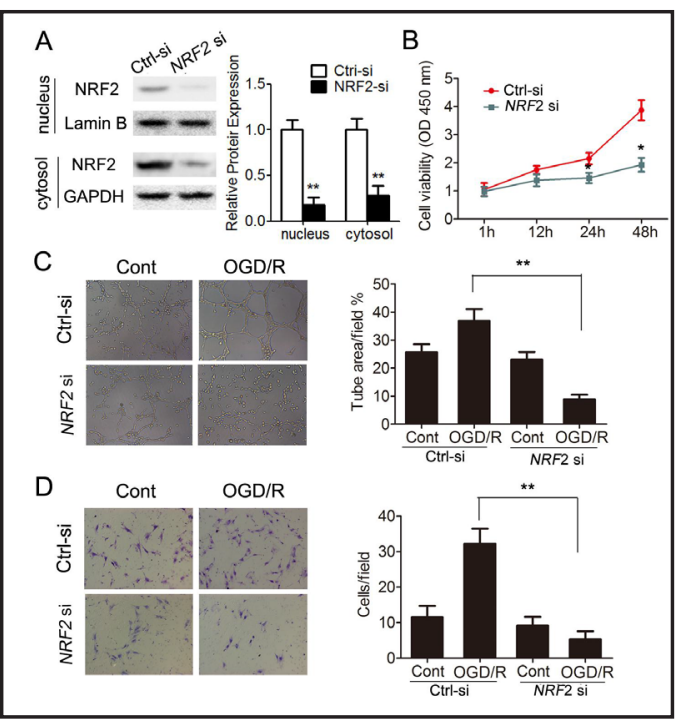

Fig. 5. Nrf2 deletion affects angiogenesis in human pulmonary microvascular endothelial cells. Human pulmonary microvascular endothelial cells (PMVECs) were transfected with or without Nrf2 siRNA for 24 hours and exposed to oxygen-glucose deprivation/reoxygenation (OGD/R) for $24 \mathrm{~h}$. (A) Nrf2 expression was monitored by western blotting. (B) Cell viability of different groups was detected by CCK-8 at different time intervals following OGD/R. (C) Angiogenesis was analysed by assays in different groups. Angiogenesis was decreased in the Nrf2 siRNA group rather than in the NS siRNA group. (D) Migration assay with non-target (Control) and Nrf2siRNA-transfected human PMVECs. $\left({ }^{*} \mathrm{p}<0.05\right.$; ${ }^{* *} \mathrm{p}<0.001$ vs. respective control).

pathway in Nrf2/Sirt1 regulation and angiogenesis in PMVECs. Western blotting, qRT-PCR, and immunofluorescence staining of NOX4 revealed that Nrf2 knockdown suppressed NOX4 expression, while the overexpression of Sirt1 upregulated NOX4 (Fig. 7A-C). Nrf2 knockdown suppressed cellular viability, capillary tube formation and cell migration in human PMVECs after OGD/R and also inhibited NOX4, HIF- $1 \alpha$ and VEGF expression. Moreover, a NOX4 knockdown in PMVECs decreased the levels of VEGF and HIF-1 $\alpha$ (Fig. 7D) and cell viability, capillary tube formation and cell migration (Fig. 7E). Taken together, these results indicate that Sirt1 induces Nrf2 upregulation and subsequently NOX4-mediated gene regulation to promote angiogenesis in human PMVECs. 
Fig. 6. Sirt1 overexpression induces Nrf2 activation and translocation into the nucleus and attenuates the reduction of survival, network formation, migration, and adhesion in PMVECs. PMVECs were transfected with or without the Sirt1 vector for $24 \mathrm{~h}$ and exposed to oxygen-glucose deprivation/reoxygenation. (A)Sirt1 expression was monitored by western blotting. (B) Immunoblot analysis of Nrf2 in the nucleus and WCE (Whole cell Extracts) at different times $(0,6,12,24 \mathrm{~h})$. (C) The level of Nrf2 was evaluated by immunofluorescence staining. (D) Cell viability of different groups was detected by CCK8. (E) Angiogenesis was detected by assays in the different groups. (F) Migration

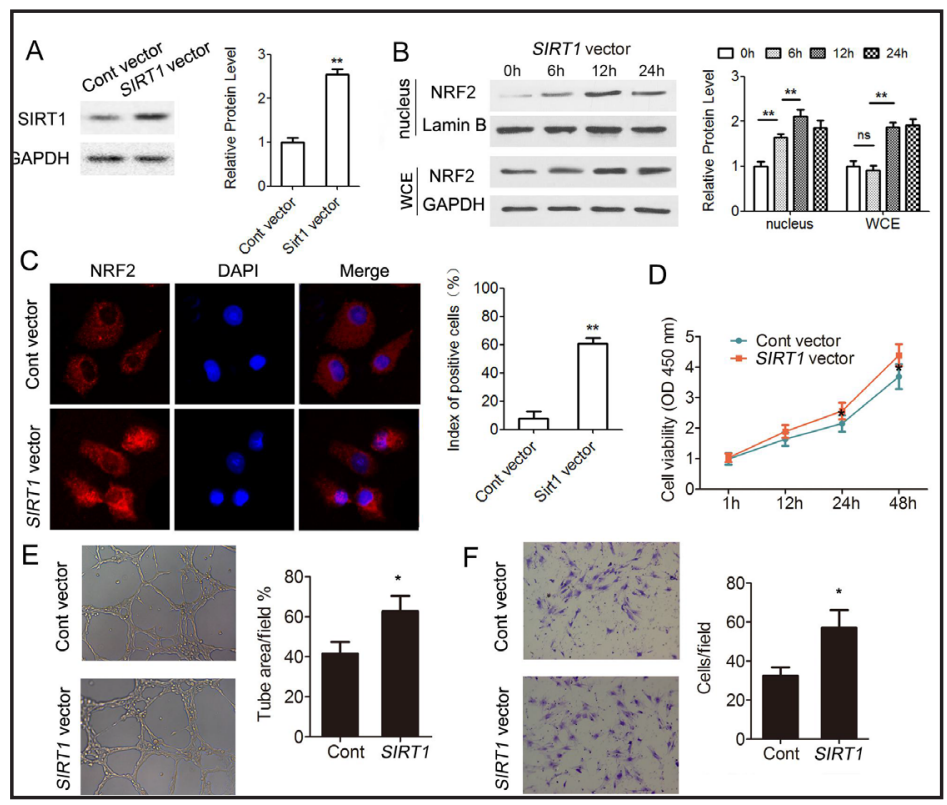
assay with non-target (Con) and Nrf2 siRNA-transfected human PMVECs. $\left(^{*} \mathrm{p}<0.05 ;{ }^{* *} \mathrm{p}<0.001\right.$ vs. respective control).

Fig. 7. Nrf2 regulates angiogenesis through NOX4-mediated gene regulation. NOX4 expression was analysed in human PMVECs, with Nrf2 knockdown or Sirt1 overexpressed, by western blotting (A), RT-PCR (B) and immunofluorescence staining (C). PMVECs were transfected with NOX4 siRNA or the control siRNA for 24 hours before oxygen-glucose deprivation/ reoxygenation. (D) HIF-1 $\alpha$ and VEGF protein levels were analysed by western blot analysis. (E) Cell viability was detected by CCK8. Angiogenesis and migration were detected by assays. ( ${ }^{*} \mathrm{p}<0.05 ;{ }^{* *} \mathrm{p}$ $<0.001$ vs. respective control).

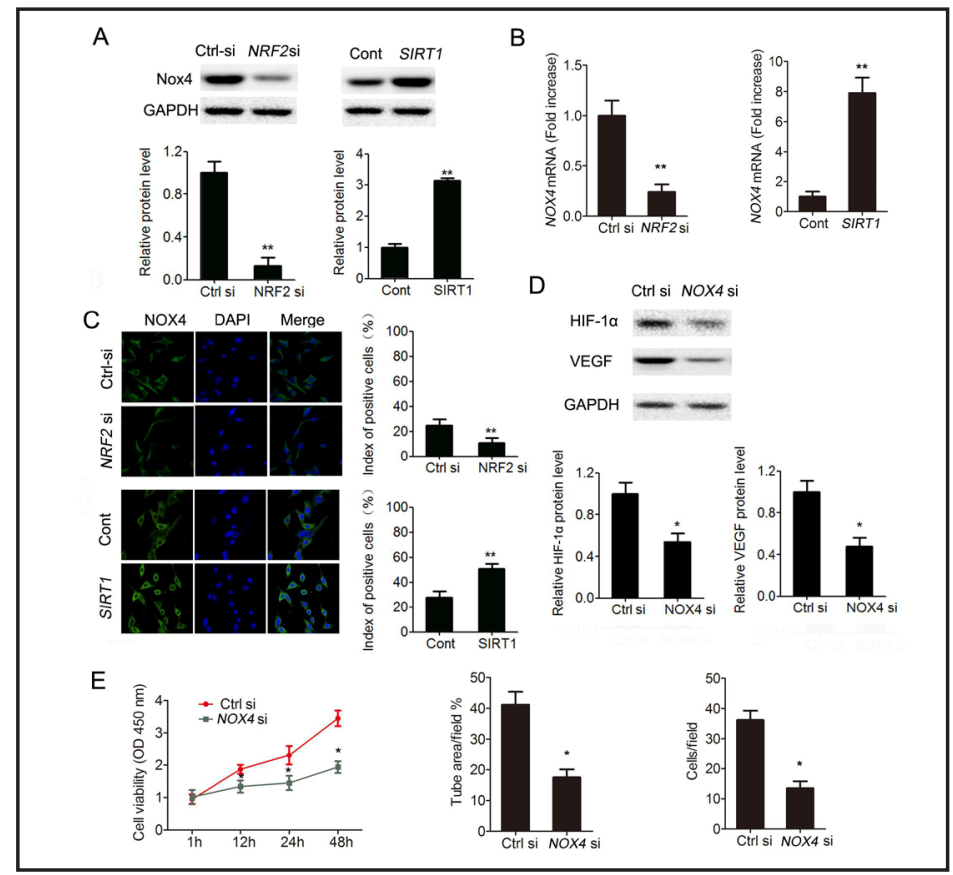

\section{Discussion}

Nrf2 has been implicated in regulating ischemic angiogenesis in a number of diseases such as cardiovascular diseases $[20,21]$ and ischemic retinopathies [11]. Nrf2 is also believed to protect a number of organs against IIR injury [22] including the lungs [23]. Furthermore, in several studies, the upregulation of Sirt1 has been found to activate Nrf2 [16, 17]. We investigated whether Nrf2 participated in the revascularization and modulation of acute lung injury after IIR in a murine model and found that Nrf2 stimulated by Sirt1 overexpression may play an important role in ischemic angiogenesis following IIR through NOX4-mediated 
gene regulation. Nrf2 upregulation and translocation into the nucleus stimulated by Sirt1 overexpression exhibited remission of histopathologic changes and enhanced endothelial cell marker CD31 expression.

The Nrf2-antioxidant response element signalling pathway is a major cellular defence against oxidative or electrophilic stress [24]. It interacts with a number of ARE-dependent genes by regulating both inducible and constitutive gene expression [25]. A greater understanding of the regulation of ARE inducible/constitutive genes could lead to the ability to control desirable qualities such as the revascularization of ischemic tissue. We found that Nrf2 knockdown in mice repressed NOX4, HIF-1 $\alpha$ and VEGF expression after IIR, and Nrf2 upregulation by Sirt1 enhances NOX4, HIF-1 $\alpha$ and VEGF expression after IIR in wild-type mice. HIF- $1 \alpha$ is involved in the transcription of VEGF, which stimulates neovascularization following hypoxia or ischemia [26]. Wang et al [27]. investigated neovascularization using a rat model of retinopathy and found that VEGF-activated NOX4 led to an interaction between VEGF-activated VEGFR2 and NOX4 that mediated endothelial cell proliferation.

Gao et al [15]. have also found that overexpression of Sirt1 may regulate endothelial cell proliferation and migration and proposed that Sirt1 could play a role in angiogenesis. They demonstrated that sphingosine kinase 1 (SPHK1) and S1P upregulates Sirt1 in human umbilical vein endothelial cells through multiple pathways including P38 MAPK, ERK and AKT signals. Zhang et al [1]. found that icariin-mediated Sirt1 had a protective effect on IIRinduced acute lung injury through the FOXO3 signalling pathway in rats. They propose that IIR may activate a cascade resulting in ROS accumulation, Sirt1 reduction, FOXO3 acetylation and MnSOD downregulation. Activation of the Nrf2/Sirt1 signalling pathway is also reported in a paraquat-induced lung injury model, where it is proposed that Sirt1 promoted the stability of Nrf2 by regulating the deacetylation and activation of the Nrf2/ARE antioxidant pathway [28].

To further investigate Nrf2/Sirt1-associated angiogenesis, we assessed the regulation of Nrf2 and Sirt1 in human PMVECs after OGD/R. We found that Sirt1 overexpression induces Nrf2 upregulation and translocation into the nucleus. Nuclear accumulation of Nrf2 is associated with enhanced Sirt1-mediated expression of NOX4, HIF-1 and VEGF. Moreover, NOX4 knockdown in PMVECs decreased the levels of VEGF and HIF-1 $\alpha$ and angiogenesis. Furthermore, Sirt1 shuttles between the cytoplasmic and nuclear compartments and regulates the gene expression involved in cell growth, cell survival and migration in response to chronic physiological and pathological cellular stresses [29]. Therefore, the Nrf2/Sirt1 regulated angiogenesis we have observed in the murine model is replicated in human pulmonary endothelial cells.

\section{Conclusion}

To summarize, we found that overexpression of Sirt1 promotes the translocation of Nrf2 to the nucleus and increases expression of NOX4, HIF-1 $\alpha$ and VEGF expression in a murine and human cell model. This led to ischemic angiogenesis in the murine model and increased cellular viability, capillary tube formation and cell migration in human cells and leaves us to conclude that Nrf2 when stimulated by Sirt1, plays an important role in angiogenesis possibly through NOX4-mediated gene regulation. Further research will lead to greater understanding of the exact mechanism involved and improve methods of managing or preventing lung injury following IIR.

\section{Acknowledgements}

This work is supported by Shanghai Pujiang Program(17PJD022) and National Natural Science Foundation of China $(81301655,81571028)$. 


\section{Cellular Physiology Cell Physiol Biochem 2018;46:781-792

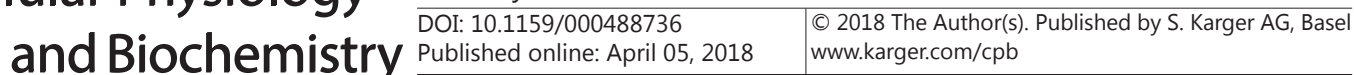

Chai et al.: Nrf2 Activation Ameliorates ALI

\section{Disclosure Statement}

The authors declare no competing financial interests.

\section{References}

1 Zhang F, Li ZL, Xu XM, Hu Y, Yao JH, Xu W, Jing HR, Wang S, Ning SL, Tian XF: Protective effects of icariinmediated Sirt1/foxo3 signaling pathway on intestinal ischemia/reperfusion-induced acute lung injury. Mol Med Rep 2015;11:269-276.

-2 Mörs K, Hörauf JA, Kany S, Wagner N, Sturm R, Woschek M, Perl M, Marzi I, Relja B: Ethanol decreases inflammatory response in human lung epithelial cells by inhibiting the canonical NF-kB-Pathway. Cell Physiol Biochem 2017;43:17-30.

-3 Liu Y, Lu J, Wang X, Chen L, Liu S, Zhang Z, Yao W: Erythropoietin-derived peptide protects against acute lung injury after rat traumatic brain injury. Cell Physiol Biochem 2017;41:2037-2044.

4 Cavriani G, Oliveirafilho RM, Trezena AG, da Silva ZL, Domingos HV, de Arruda MJ, Jancar S, Tavares de Lima W: Lung microvascular permeability and neutrophil recruitment are differently regulated by nitric oxide in a rat model of intestinal ischemia-reperfusion. Eur J Pharmacol 2004;494:241-249.

5 Mura M, Andrade CF, Han B, Seth R, Zhang Y, Bai X-H, Waddell TK, Hwang D, Keshavjee S, Liu M: Intestinal $\mathrm{i} / \mathrm{r}$-induced acute lung injury and oncotic cell death in multiple organs. Shock 2007;28:227-38.

6 Tai W, Xu Y, Ding J, Wu H, Du M, Qu X, Gao L, Li J, Dong Z: Fibrocytesameliorate acute lung injury by decreasing inflammatory cytokine and chemokine levels and reducing neutrophil accumulation in the lung. Cell Physiol Biochem 2017;44(4):1526-1536.

7 Kensler TW, Wakabayashi N, Biswal S: Cell survival responses to environmental stresses via the keap1Nrf2-are pathway. Annu Rev Pharmacol Toxicol 2007;47:89-116.

8 Shen CC, Chen B, Gu JT, Ning JL, Chen L, Zeng J, Yi B, Lu KZ: The angiogenic related functions of bone marrow mesenchymal stem cells are promoted by cbdl rat serum via the akt/Nrf2 pathway. Exp Cell Res 2016;344:86-94.

-9 Zhang HX, Liu SJ, Tang XL, Duan GL, Ni X, Zhu XY, Liu YJ, Wang CN: H2S attenuates LPS-induced acute lung injury by reducing oxidative/nitrative stress and inflammation. Cell Physiol Biochem 2016;40:1603-1612.

10 Wei Y, Gong J, Xu Z, Duh EJ: Nrf2 promotes reparative angiogenesis through regulation of nadph oxidase-2 in oxygen-induced retinopathy. Free Radic Biol Med 2016;99:234-243.

11 Wei Y, Gong J, Xu Z, Thimmulappa RK, Mitchell KL, Welsbie DS, Biswal S, Duh EJ: Nrf2 in ischemic neurons promotes retinal vascular regeneration through regulation of semaphorin 6a. Proc Natl Acad Sci U S A 2015;112:E6927-6936.

12 Cho HY, Kleeberger SR: Nrf2 protects against airway disorders. Toxicol Appl Pharmacol 2010;244:43-56.

13 Xu X, Zhu Q Zhang R, Wang Y, Niu F, Wang W, Sun D, Wang A: ITRAQ-Based proteomics analysis of acute lung injury induced by oleic acid in Mice. Cell Physiol Biochem 2017;44:1949-1964.

14 Pendyala S, Moitra J, Kalari S, Kleeberger SR, Zhao Y, Reddy SP, Garcia JG, Natarajan V: Nrf2 regulates hyperoxia-induced nox4 expression in human lung endothelium: Identification of functional antioxidant response elements on the nox4 promoter. Free Radic Biol Med 2011;50:1749-1759.

15 Gao Z, Wang H, Xiao FJ, Shi XF, Zhang YK, Xu QQ, Zhang XY, Ha XQ, Wang LS: Sirt1 mediates sphk1/s1pinduced proliferation and migration of endothelial cells. Int J Biochem Cell Biol 2016;74:152-160.

$16 \mathrm{Gu}$ L, Tao X, Xu Y, Han X, Qi Y, Xu L, Yin L, Peng J: Dioscin alleviates bdl- and dmn-induced hepatic fibrosis via Sirt1/Nrf2-mediated inhibition of p38 mapk pathway. Toxicol Appl Pharmacol 2016;292:19-29.

17 Zhou Z, You Z: Mesenchymal stem cells alleviate LPS-induced acute lung injury in mice by MiR-142a-5pcontrolled pulmonary endothelial cell autophagy. Cell Physiol Biochem 2016;38:258-66.

18 Ding YW, Zhao GJ, Li XL, Hong GL, Li MF, Qiu QM, Wu B, Lu ZQ: Sirt1 exerts protective effects against paraquat-induced injury in mouse type ii alveolar epithelial cells by deacetylating Nrf2 in vitro. Int J Mol Med 2016;37:1049-1058.

19 Ding M, Lei J, Han H, Li W, Qu Y, Fu E, Fu F, Wang X: Sirt1 protects against myocardial ischemia-reperfusion injury via activating enos in diabetic rats. Cardiovasc Diabetol 2015;14:143. 


\section{Cellular Physiology Cell Physiol Biochem 2018;46:781-792 and Biochemistry Published 1159/000488736 $2018 \quad \begin{aligned} & \text { DO 2018 The Author(s). Published by S. Karger AG, Basel } \\ & \text { www.karger.com/cpb }\end{aligned}$ \\ Chai et al.: Nrf2 Activation Ameliorates ALI}

20 Shimizu Y, Nicholson CK, Lambert JP, Barr LA, Kuek N, Herszenhaut D, Tan L, Murohara T, Hansen JM, Husain A, Naqvi N, Calvert JW: Sodium sulfide attenuates ischemic-induced heart failure by enhancing proteasomal function in an Nrf2-dependent manner. Circ Heart Fail 2016;9:e002368.

-21 Hou JY, Liu Y, Liu L, Li XM: Protective effect of hyperoside on cardiac ischemia reperfusion injury through inhibition of er stress and activation of Nrf2 signaling. Asian Pac J Trop Med 2016;9:76-80.

-22 Li W, Suwanwela NC, Patumraj S: Curcumin by down-regulating nf-kb and elevating Nrf2, reduces brain edema and neurological dysfunction after cerebral i/r. Microvasc Res 2016;106:117-127.

-23 Meng QT, Cao C, Wu Y, Liu HM, Li W, Sun Q, Chen R, Xiao YG, Tang LH, Jiang Y, Leng Y, Lei SQ, Lee CC, Barry DM, Chen X, Xia ZY: Ischemic post-conditioning attenuates acute lung injury induced by intestinal ischemiareperfusion in mice: Role of Nrf2 Lab Invest 2016;96:1087-1104.

24 Nguyen T, Nioi P, Pickett CB: The Nrf2-antioxidant response element signaling pathway and its activation by oxidative stress. J Biol Chem 2009;284:13291-13295.

-25 Nguyen T, Sherratt PJ, Nioi P, Yang CS, Pickett CB: Nrf2 controls constitutive and inducible expression of are-driven genes through a dynamic pathway involving nucleocytoplasmic shuttling by keap1 J Biol Chem 2005;280:32485-32492.

-26 Yu X, Lin Q, Qin X, Ruan Z, Zhou J, Lin Z, Su Y, Zheng J, Liu Z. ACE2 antagonizes VEGFa to reduce vascular permeability during acute lung injury. Cell Physiol Biochem 2016;38:1055-62.

27 Wang H, Yang Z, Jiang Y, Hartnett ME: Endothelial NADPH oxidase 4 mediates vascular endothelial growth factor receptor 2-induced intravitreal neovascularization in a rat model of retinopathy of prematurity. Mol Vis 2014; 20:231-41.

28 Ding YW, Zhao GJ, Li XL, Hong GL, Li MF, Qiu QM, Wu B, Lu ZQ. SIRT1 exerts protective effects against paraquat-induced injury in mouse type II alveolar epithelial cells by deacetylating NRF2 in vitro. Int J Mol Med. 2016;37:1049-1058.

-29 Potteti HR, Rajasekaran S, Rajamohan SB, Tamatam CR, Reddy NM, Reddy SP: Sirtuin 1 promotes hyperoxia-induced lung epithelial cell death independent of nf-e2-related factor 2 activation. Am J Respir Cell Mol Biol 2016;54:697-706. 